\title{
EL TERRORISMO EN LA JURISPRUDENCIA DEL TRIBUNAL PENAL INTERNACIONAL PARA LA ANTIGUA YUGOSLAVIA
}

\author{
Soledad Inés Castro \\ Secretaria Letrada de la Corte Suprema de Justicia de la Nación \\ Contacto:doc_soledadcastro@yahoo.com.ar
}

Recibido: 28 de agosto de 2020 Aprobado: 20 de septiembre de 2020

Para citar este artículo:

Castro, S. I. (2021). "E1 terrorismo en la jurisprudencia del Tribunal Penal Internacional para la Antigua Yugoslavia”. Prudentia Iuris, N. 91, pp. 73-100 DOI: https://doi.org/10.46553/prudentia.91.2021.pp.73-100

Resumen: El Estatuto del Tribunal Penal Internacional para la Antigua Yugoslavia no contemplaba expresamente una definición de terrorismo, ni lo incluía entre los crímenes bajo su jurisdicción. Ello no impidió que el tribunal desarrollara jurisprudencia propia en la materia. El presente trabajo se propone revisar la evolución jurisprudencial del Tribunal Penal Internacional para la Antigua Yugoslavia relativa a la caracterización y criminalización del terrorismo en contextos de conflicto armado, a partir del paradigmático caso "Galić", analizando sus antecedentes y su aplicación posterior, para reflexionar -en última instancia- sobre la gravitación de estos desarrollos jurisprudenciales en la más amplia tarea de definir al terrorismo en el Derecho Internacional.

Palabras clave: Terrorismo, Crímenes de Guerra, Derecho Internacional Humanitario, Tribunales Internacionales. 


\title{
The International Criminal Tribunal for the Former Yugoslavia and its Case Law on Terrorism
}

\begin{abstract}
The ICTY's Statute did not explicitly establish its jurisdiction over the crime of terror nor did it include a definition. Nonetheless, such circumstances did not prevent the tribunal from developing its own jurisprudence regarding the issue of terrorism. This article aims to compile and exam the ICTY's case law pertaining to the evolution of the definition and criminalization of terrorism. With the focus on the case of "Prosecutor v. Stanislav Galić", it reviews its doctrine and the jurisprudence before and after it regarding those specific matters. In the final remarks, this relevant jurisprudence is assessed among other endeavors made to define terrorism in international law.
\end{abstract}

Keywords: Terrorism, War Crimes, International Humanitarian Law, International Tribunals.

\section{Il terrorismo in la giurisprudenza del Tribunale Penale Internazionale per l'ex Iugoslavia}

Sommario: Lo statuto del Tribunale Penale Internazionale per l'ex Jugoslavia non prevedeva espressamente una definizione di terrorismo, né lo includeva tra i crimini che rientrano nella sua giurisdizione. Ciò non ha impedito alla Corte di sviluppare una propria giurisprudenza in materia. Il presente lavoro intende riesaminare l'evoluzione giurisprudenziale del Tribunale penale internazionale per l'ex Iugoslavia relativa alla caratterizzazione e alla criminalizzazione del terrorismo in contesti di conflitto armato, a partire dal paradigmatico caso "Galić", analizzandone il contesto e la successiva applicazione, per riflettere -in ultima istanza-, sulla gravitazione di questi sviluppi giurisprudenziali nel più ampio compito di definire il terrorismo nel diritto internazionale.

Parole chiave: Terrorismo, Crimini di Guerra, Diritto Umanitario Internazionale, Tribunali Internazionali. 


\section{Introducción*}

Dentro del sistema de cortes internacionales y, más precisamente, aquellas con jurisdicción penal individual, el Tribunal Penal Internacional para la Antigua Yugoslavia (en adelante, TPIY) fue el primero de los tribunales "ad hoc" creado por el Consejo de Seguridad de Naciones Unidas". En su Estatuto, quedó delimitada su competencia, habilitada a juzgar a los presuntos responsables de violaciones del Derecho Internacional Humanitario cometidas a partir del $1^{\circ}$ de enero de 1991 en el territorio de la ex-Yugoslavia, por los siguientes crímenes: a) infracciones graves a los Convenios de Ginebra de 1949; b) violaciones de las leyes o costumbres de la guerra; c) genocidio y d) crímenes de lesa humanidad (cfr. arts. $1^{\circ}$ a $5^{\circ}$ y cctes. del Estatuto del TPIY) ${ }^{2}$.

Como ya se destacó, el citado Estatuto no contenía una definición de terrorismo como crimen autónomo ${ }^{3}$, no describía sus elementos típicos y tampoco lo incluía expresamente entre las graves violaciones a los Convenios de Ginebra ni como violación a las leyes o usos de la guerra, enumeradas en su texto. Estas circunstancias no resultaron obstáculo para que, tanto en primera como en segunda instancia, el TPIY condenara a Stanislav Galić -entre otros delitos- por el crimen de terrorismo. El caso "Galić" se

* El presente trabajo se desarrolló en el marco del Proyecto IUS "El Terrorismo Internacional. Desafíos actuales del Derecho Penal local e internacional" (2019-2021) de la Pontificia Universidad Católica Argentina, dirigido por la Mg. Sofía J. Danessa. Contó con la inestimable colaboración de las abogadas Emily Montenegro Seadi y Andrea Aguirre, miembros del citado proyecto.

1 S.C. Res. 808, 1, U.N. Doc. S/RES/808 (Feb. 22, 1993) (creación del TPIY).

2 S.C. Res. 827, U.N. Doc. S/RES/827 (May 25, 1993) (Estatuto del TPIY). Enmiendas por: S.C. Res. 1166, U.N. Doc. S/RES/1166 (May 13, 1998); S.C. Res. 1329, U.N. Doc. S/RES/1329 (Nov. 30, 2000); S.C. Res. 1411, U.N. Doc. S/RES/1411 (May 17, 2002); S.C. Res. 1431, U.N. Doc. S/RES/1431 (Aug. 14, 2002); S.C. Res. 1481, U.N. Doc. S/RES/1481 (May 19, 2003); S.C. Res. 1597, U.N. Doc. S/RES/1597 (April 20, 2005); S.C. Res. 1660, U.N. Doc. S/RES/1660 (Feb. 28, 2006); S.C. Res. 1837, U.N. Doc. S/RES/1837 (Sept. 29, 2008); S.C. Res. 1877, U.N. Doc. S/ RES/1877 (July 7, 2009); S.C. Res. 1931, U.N. Doc. S/RES/1931 (June 29, 2010); S.C. Res. 1993, U.N. Doc. S/RES/1993 (June 29, 2011); S.C. Res. 2306, U.N. Doc. S/RES/2306 (Sept. 6, 2016). El TPIY investigó y enjuició a 161 individuos por serias violaciones al Derecho Internacional humanitario cometidas en el territorio de la Antigua Yugoslavia, ver: https://www.icty.org/ sid/24 (disponible al 9/8/2020).

3 Esta circunstancia constituye una diferencia respecto del Tribunal Penal Internacional para Ruanda (TPIR) y del Tribunal Especial para Sierra Leona (TESL), los cuales expresamente incorporan a los actos de terrorismo entre los crímenes de su competencia (cfr. art. $4^{\circ}$, inc. d del Estatuto del TPIR y art. $3^{\circ}$, incs. d, h, del Estatuto del TESL). Cfr: S.C. Res. 955, U.N. Doc. S/RES/955 (Nov. 8, 1994) (Estatuto del TPIR); S.C. Res. 1315, U.N. Doc. S/RES/1315 (Aug. 14, 2000) (Estatuto del TESL). 
convirtió, así, en el primer precedente internacional en que se condenó por terrorismo, como un crimen de guerra autónomo.

El presente trabajo consta de cuatro secciones. En la primera, se revisa un conjunto de casos que precedieron al fallo "Galić, en los que las cámaras del TPIY abordaron el juzgamiento de hechos que involucraron, en alguna medida, la acción o la pretensión de aterrorizar a civiles en particular o a la población civil en general. En la segunda sección y respetando la cronología jurisprudencial, se desarrollan los aspectos centrales del caso "Galić", tanto en lo relativo a la caracterización del crimen de terrorismo a los fines de la competencia material del tribunal (apartado 2.a) como a la figura penal y sus elementos típicos (apartado 2.b). En la tercera sección, se examina el alcance que la doctrina del fallo "Galić" en materia de terrorismo tuvo en la jurisprudencia posterior del propio TPIY. Finalmente, se realizan unas breves reflexiones sobre el modo de interpretar estos aportes jurisprudenciales a la luz de la tarea colectiva de caracterizar y criminalizar al terrorismo.

\section{Antecedentes en el TPIY: Antes de "Galić"}

En los pronunciamientos del tribunal que precedieron al caso "Galić", se advierten alusiones al terror en la caracterización de situaciones y prácticas correspondientes a los sucesos sometidos a juicio y, en ciertos casos, se observa que las cámaras intervinientes criminalizaron actos y políticas con contenido terrorista por entender que configuraban alguno de los crímenes incluidos en la competencia del tribunal. A continuación, se compila una breve reseña.

En Prosecutor $v$. Tadic, testigos y víctimas se refirieron reiteradamente a la "atmósfera de terror" en el marco de la cual tuvieron lugar ciertos hechos o se cometieron ciertos actos ${ }^{4}$. Entre los múltiples cargos de la acusación, la fiscalía atribuyó a las fuerzas serbias el haber sometido a croatas y musulmanes -dentro y fuera de los campos de detención- a una campaña de terror que incluía asesinatos, tortura, ataques sexuales y otros abusos físicos y psicológicos, y aportó testimonios que daban cuenta de una evidente política destinada a aterrorizar a la población civil no serbia, con fundamento discriminatorio ${ }^{5}$. El tribunal de juicio mencionó al terrorismo como uno

4 "[...] the atmosphere of terror which prevailed in the camp [...]". Prosecutor $v$. Dusko Tadic a/k/a/ "DULE", IT-94-1-T, Trial Chamber, Opinion and Judgment, 7 May 1997, para. 164.

5 "[...] subjected Muslims and Croats inside and outside the camps to a campaign of terror which included killings, torture, sexual assaults, and other physical and psychological abuse [...] A policy to terrorize the non-Serb civilian population [...] on discriminatory 
de los medios para la consecución del plan de limpieza étnica directamente relacionado al conflicto armado ${ }^{6}$ y el terror infundido a las víctimas fue considerado como una circunstancia agravante en la determinación del monto de las penas ${ }^{7}$.

En Prosecutor v. Blaskic, se aludió al terrorismo en la descripción de algunos de los hechos que conformaron el objeto procesal del juicio. Por ejemplo, un atentado con explosivos fue considerado -tanto en primera como en segunda instancia- como un acto terrorista que configuraba, a su vez, un crimen contra la humanidad ${ }^{8}$. También se sostuvo que fuerzas militares habían bombardeado y asesinado con el fin de aterrorizar a la población civil, para lograr que abandonaran ciertas áreas y no regresaran y se mencionó la "atmósfera de terror" que existía en distintos lugares de detención ${ }^{9}$.

En Prosecutor v. Dario Kordi et Mario Cerkez, el tribunal de juicio identificó ciertos actos de terrorismo dentro de los hechos pesquisados, pero descartó los sucesos respecto de los cuales no existía evidencia que sustentara la atribución de responsabilidad a los acusados ${ }^{10}$.

En Prosecutor v. Radislav Krstić, el tribunal de juicio concluyó que la campaña de bombardeo a Srebrenica estaba dirigida a crear una crisis humanitaria, aterrorizando a la población bosnio-musulmana para lograr que, por miedo, abandonaran el lugar. Asimismo, entendió que los sucesos de Potočari estuvieron enmarcados en una "campaña de terror"; es más, que allí se cometieron "crímenes de terror". En concreto, el tribunal de juicio concluyó que Radislav Krstić era penalmente responsable y, en lo que aquí interesa, lo condenó por acciones destinadas a aterrorizar a la población civil bosnio-musulmana, configurativas de actos persecutorios y, como tales,

grounds [...]". Prosecutor v. Dusko Tadic a/k/a/ "DULE”, IT-94-1-T, Trial Chamber, Opinion and Judgment, 7 May 1997, paras. 377, 472.

6 "[...] such treatment effected the objective of the Republika Srpska to ethnically cleanse, by means of terror, killings or otherwise, the areas of the Republic of Bosnia and Herzegovina controlled by Bosnian Serb forces [....", en: Prosecutor v. Dusko Tadic a/k/a/ "DULE", IT-94-1-T, Trial Chamber, Opinion and Judgment, 7 May 1997, para. 575.

7 Prosecutor v. Dusko Tadic a/k/a/ "DULE", IT-94-1-T, Trial Chamber, Sentencing Judgment, 14 July 1997, para. 56.

8 Prosecutor v. Tihomir Blaskic, IT-95-14-T, Trial Chamber, Judgment, 3 March 2000, para. 505, 511. Prosecutor v. Tihomir Blaskic, IT-95-14-T, Appeals Chamber, Judgment, 29 July 2004, para. 447.

9 Prosecutor v. Tihomir Blaskic, IT-95-14-T, Trial Chamber, Judgment, 3 March 2000, paras. $630,695,700,732$.

10 "However, there is no evidence to connect either of the accused with this action, which is as consistent with freelance terrorist activity by any of a number of people as it is as part of a concerted plan of ethnic cleansing [...]", en: Prosecutor v. Dario Kordi et Mario Cerkez, IT-95-14/2-T, Trial Chamber, Judgment, 26 February 2001, para 664, 675. Prosecutor v. Dario Kordi et Mario Cerkez, IT-95-14/2-T, Appeals Chamber, Judgment, 17 December 2004, para 464. 
crímenes contra la humanidad ${ }^{11}$. Si bien la cámara de apelaciones modificó aspectos vinculados al tipo de participación y responsabilidad, reduciendo la pena, la caracterización del terrorismo quedó inalterada ${ }^{12}$.

En relación con estos hechos y en el marco del proceso Prosecutor $v$. Momir Nikolić, el acusado reconoció su culpabilidad en la comisión de actos de persecución, entre los que se encontraban las acciones destinadas a aterrorizar a la población civil bosnio-musulmana en Srebrenica y Potočari, calificados como crímenes de lesa humanidad ${ }^{13}$.

En Prosecutor v. Zejnil Delalić, Zdravko Mucić also known as "Pavo", Hazim Delić, Esad Landžo also known as "Zenga", conocido como el caso del campo de prisioneros de Čelebići, el tribunal de juicio estableció que los detenidos en ese campo fueron sometidos, además, a una "atmósfera de terror psicológico", a causa del miedo constante a ser víctimas de los abusos físicos que presenciaban. Se concluyó que las acciones tendientes a crear y sostener esa atmósfera de terror configuraron los crímenes de tratos crueles y de intencionada causación de gran sufrimiento o lesiones graves a la integridad física o la salud; es decir, graves violaciones a las leyes y prácticas de la guerra y a los Convenios de Ginebra, respectivamente. Con distinto grado de participación y responsabilidad penal, se condenó a los acusados,

11 "[...] the shelling of Srebrenica [...] was calculated to terrify the Bosnian Muslim population and to drive them out of Srebrenica town [...] the events that forced the terrorized civilian population of Srebrenica to flee the town in fear of their lives and move toward Potocari [...] the impact of the shelling upon the terrorized Bosnian Muslim residents of Srebrenica [...] The atmosphere of terror in which the evacuation was conducted proves, conversely, that the transfer was carried out in furtherance of a well organized policy whose purpose was to expel the Bosnian Muslim population from the enclave [...]"; "[...] the Bosnian Muslim refugees taking shelter in and around the compound were subjected to a terror campaign comprised of threats, insults, looting and burning of nearby houses, beatings, rapes, and murders [...] The entire situation in Potočari has been depicted as a campaign of terror [...]"; "[...] The Trial Chamber characterizes the humanitarian crisis, the crimes of terror and the forcible transfer of the women, children and elderly at Potočari as constituting crimes against humanity, that is, persecution and inhumane acts [...]"; "[...] on the basis of the [...] crimes of terror at Potočari [...] Trial Chamber enters convictions [...] for charges of persecution [...] terrorizing the civilian population [....'. Prosecutor v. Radislav Krstić, IT-98-33-T, Trial Chamber, Judgment, 2 August 2001, paras. (122, 125, 335, 527); (41, 44, 46, 150, 153, 292, 364, 517); (606/607); 653, $661,670,671,677$.

12 Prosecutor v. Radislav Krstić, IT-98-33-A, Appeals Chamber, Judgment, 19 April 2004.

13 "[...] During the time-period that the Bosnian Muslim refugee population from Srebrenica was in and around Potočari, members of the VRS terrorized the population [...] this took the form of intimidation and abuse, with the purpose of compelling the Muslim population to get on the buses and trucks to Kladanj [...]", Prosecutor v. Momir Nikolić, IT-02-60/1-S, Trial Chamber, Sentencing Judgment, 2 December 2003, paras. 31, 38, 174. 
entre otros crímenes, por estos hechos ${ }^{14}$. Es preciso destacar, aquí, que la tipificación de esos actos fue confirmada, a su turno, por la cámara de apelaciones ${ }^{15}$.

$\mathrm{Al}$ respecto, cabe recordar que, por hechos menos gravosos a los antes narrados, en Prosecutor v. Zlatko Aleksovski también se dictó sentencia condenatoria (agravada en segunda instancia) por abuso psicológico de prisioneros como modalidad de maltrato, caracterizado como "terror psicológico"16.

En suma, a la luz de este primer conjunto de casos, las acciones o prácticas sometidas a juicio del tribunal que, de algún modo, importaban aterrorizar a civiles en particular o a la población civil en general, fueron criminalizadas en distintas figuras. Así, el terrorismo fue sancionado como circunstancia agravante de pena, como acto persecutorio con fundamento discriminatorio, configurativo de crímenes de lesa humanidad, como trato cruel violatorio de las leyes y usos de la guerra e, incluso, como grave infracción a los Convenios de Ginebra, en tanto acto intencionado de causar gran sufrimiento o lesiones graves a la integridad física o a la salud.

En otras palabras, del análisis de la jurisprudencia temprana del tribunal se desprende que, si bien el terrorismo no fue reprimido mediante la aplicación de un tipo penal específico, el contenido terrorista de las conductas juzgadas no quedó impune, sino que fue penalmente atribuido como agravante o como acción constitutiva de crímenes expresamente previstos

14 "[...] the detainees in the Čelebici prison-camp were continuously witnessing the most severe physical abuse being inflicted on defenseless victims [...] by their exposure to these conditions, the detainees were compelled to live with the ever-present fear of being killed or subjected to physical abuse [...] Through the frequent cruel and violent deeds committed in the prison-camp, aggravated by the random nature of these acts and the threats made by guards, the detainees were thus subjected to an immense psychological pressure which may accurately be characterized as an atmosphere of terror [...]"; "[...] the chronic physical deprivation and the constant fear prevailing in the Čelebici prison-camp caused serious mental and physical suffering to the detainees. Moreover, for the purposes of the offence of cruel treatment, exposure to these conditions clearly constituted an attack upon the human dignity of the detainees. Accordingly, on the basis of the foregoing evidence, the Trial Chamber finds that the creation and maintenance of an atmosphere of terror in the Čelebici prison-camp, by itself and $\boldsymbol{a}$ fortiori, together with the deprivation of adequate food, water, sleeping and toilet facilities and medical care, constitutes the offence of cruel treatment under article 3 of the Statute, and willfully causing great suffering or serious injury to body or health under article 2 of the Statute [...]", Prosecutor v. Zejnil Delalić, Zdravko Mucić also known as "Pavo", Hazim Delić, Esad Landžo also known as "Zenga”, IT-96-21-T, Trial Chamber, Judgment, 16 November 1998, paras. 1086/91, 1073, 1075, 1119, 1121/23, 1237, 1253, 1266, 1272, 1276, entre otros.

15 Prosecutor v. Zejnil Delalić, Zdravko Mucić also known as "Pavo", Hazim Delić, Esad Landžo also known as "Zenga”, IT-96-21-A, Appeals Chamber, Judgment, 20 February 2001.

16 Prosecutor v. Zlatko Aleksovski, IT- 95-14/1-T, Judgement, 25 June 1999. Prosecutor v. Zlatko Aleksouski, IT-95-14/1-A, Judgement, 24 March 2000, para. 183. 
en la competencia del TPIY. Estos primeros precedentes fueron expresamente sopesados por el tribunal a la hora de pronunciarse en el caso "Galić"17.

\section{Prosecutor v. Stanislav Galić}

El trayecto jurisprudencial previamente analizado condujo al caso "Galić", en el que, por primera vez, una corte internacional dictó sentencia condenatoria por terrorismo concebido como crimen de guerra autónomo ${ }^{18}$.

En lo que aquí interesa, el TPIY llevó adelante el juicio contra el general serbo-bosnio Stanislav Galić por su rol como comandante del Cuerpo de Sarajevo-Romanija (SRK) del ejército de la República de Srpska (VRS), en el sitio y el asedio a Sarajevo durante la guerra de Bosnia. Galić fue condenado por terrorismo como crimen de guerra y por crímenes contra la humanidad (actos inhumanos y homicidios), de conformidad con lo previsto en los artículos $3^{\circ}$ y $5^{\circ}$ del Estatuto ${ }^{19}$, por haber dirigido, entre septiembre de 1992 y agosto de 1994, una campaña de ataques ejecutados mediante bombardeos y francotiradores en la ciudad de Sarajevo y sus alrededores -entonces, mayormente, bajo dominio del ejército de la República de Bosnia-Herzegovina $(\mathrm{ABiH})-$, con el propósito principal de propagar terror en la población civil, ocasionando, además, muerte o lesiones en numerosas víctimas ${ }^{20}$.

La postura mayoritaria de la cámara de juicio impuso una pena de veinte años de prisión. Discrepó de la disidencia en cuestiones relativas a la plataforma fáctica (principalmente, sobre la efectiva acreditación de la campaña de ataques), a la responsabilidad penal del condenado ${ }^{21} \mathrm{y}$, espe-

17 Prosecutor v. Stanislav Galić, IT-98-29-T, Trial Chamber, Judgement, 5 December 2003, para. 66 .

18 Paredi, L. (June 2015). "The war crime of terror: an analysis of international jurisprudence", International Crimes Database (ICD) Brief 11, 1-2.

19 Prosecutor v. Stanislav Galić, IT-98-29-T, Trial Chamber, Judgement, 5 December 2003. La decisión se adoptó por la mayoría conformada por los magistrados Alphons Orie y Amin El Mahdi y la disidencia parcial del juez Rafael Nieto-Navia.

20 "[...] a professional army conducted a campaign of unrelenting violence against the inhabitants of a European city so as to reduce them to a state of medieval deprivation in which they were in constant fear of death [...] there was nowhere safe for a Sarajevan, not at home, at school, in a hospital, from deliberate attack [...]", Prosecutor v. Stanislav Galić, IT-98-29-T, Trial Chamber, Judgement, 5 December 2003, Prosecution Opening Statement, T. 562-3.

$21 \mathrm{Si}$ bien hubo coincidencia respecto del efectivo control del general Galić sobre las acciones de su tropa, la disidencia no consideró probado que el general hubiera ordenado ataques a la población civil de Sarajevo, por lo que consideró que solo cabía responsabilizarlo por no haber prevenido o sancionado los crímenes cometidos por las fuerzas a su cargo (cfr. arts. 7.1 y 7.3 del Estatuto). 
cialmente, en lo atinente a jurisdicción del tribunal respecto al crimen de terrorismo.

La sentencia de primera instancia fue recurrida por la defensa de Galić que, entre otros agravios, cuestionó la jurisdicción del tribunal respecto del crimen de terrorismo y los fundamentos esgrimidos para afirmarla, así, como la caracterización del aludido crimen y sus elementos típicos. Por su parte, la fiscalía impugnó el monto de la pena. Finalmente, a su turno, la cámara de apelaciones del TPIY resolvió, también por mayoría, desestimar la apelación de la defensa, hacer lugar al recurso de la fiscalía, y condenar a Stanislav Galić a prisión perpetua ${ }^{22}$.

A continuación, se analizarán los dos temas centrales del caso para este estudio: a) la jurisdicción del tribunal respecto del crimen de terrorismo y b) sus elementos típicos.

\section{2.a. La jurisdicción del TPIY respecto del crimen de terrorismo}

Tanto la cámara de juicio como la de apelaciones se pronunciaron, por mayoría, en favor de reconocer la jurisdicción del tribunal para entender en casos de terrorismo como un crimen de guerra autónomo. Seguidamente, se analizan los argumentos mayoritarios y las disidencias en ambas instancias.

En primer lugar, corresponde destacar que la mayoría del tribunal de juicio circunscribió su pronunciamiento a los hechos del caso, conforme descriptos en la acusación fiscal, es decir, expresamente aclaró que no le correspondía decidir si el tribunal tenía jurisdicción sobre terrorismo en general, sino que su tarea se limitaba a establecer si la corte era competente para juzgar bajo la figura de terrorismo a la acción específica de matar y herir civiles en el marco de un conflicto armado, ejecutada con la intención de aterrorizar a la población civil ${ }^{23}$.

En segundo lugar, esa misma mayoría reconoció que el crimen de terrorismo no ha sido singular y universalmente definido en Derecho Internacional y especificó que si el TPIY asumía jurisdicción lo hacía, en cualquier caso, respecto al régimen legal específicamente desarrollado para contextos de conflicto armado, expresado en el IV Convenio de Ginebra y ambos

22 Prosecutor v. Stanislav Galić, IT-98-29-T, Appeals Chamber, Judgement, 30 November 2006. La decisión se adoptó con los votos de los magistrados Fausto Pocar, Mohamed Shahabuddeen, Mehmet Güney, Theodor Meron y Wolfgang Schomburg.

23 Prosecutor v. Stanislav Galić, IT-98-29-T, Trial Chamber, Judgement, 5 December 2003, para. 87. 
Protocolos Adicionales, y no incluía los esquemas normativos creados para combatir distintos tipos de "terrorismo político"24.

En tercer lugar, para ser considerada una de las violaciones a las leyes y usos de la guerra, de aquellas previstas en el artículo $3^{\circ}$ del Estatuto ${ }^{25}$, y así afirmar la jurisdicción del tribunal, se entendió que la conducta imputada debía cumplir con dos prerrequisitos comunes a ese tipo de violaciones y cuatro condiciones, todos ellos sistematizados por el propio TPIY en el precedente Prosecutor v. Duško Tadić.

Sin disidencias, se estimaron cumplidos los dos prerrequisitos del artículo $3^{\circ}$ del Estatuto, dado que: 1) la conducta había sido cometida en el marco de un conflicto armado, y 2) se encontraba vinculada al mismo ${ }^{26}$.

Contrariamente, en el análisis respecto del cumplimiento de lo que se conoce como "las cuatro condiciones de Tadić", hubo discrepancias. Las cuatro "condiciones" para considerar que la acción juzgada configura una violación a las leyes y usos de la guerra previstas estatutariamente, consisten en: 1) la conducta imputada debe configurar una infracción a una regla de DIH; 2) la regla infringida debe ser de naturaleza consuetudinaria o, si pertenece al Derecho Convencional, debe cumplir con ciertas condiciones específicas; 3) la violación a la norma reconocida en el Derecho Internacional Humanitario debe ser "grave", debe infringir una norma que proteja valores importantes y su incumplimiento debe aparejar serias consecuencias para

24 "[...] The Majority is aware that several international instruments exist outlawing 'terrorism' in various forms. The Majority necessarily limits itself to the legal regime that has been developed with reference to conventional armed conflict [...] the Majority proceeds on the understanding that the present case will have a basis, if at all, in the legal regime of the Geneva Conventions and the Additional Protocols and not in international efforts directed against 'political' varieties of terrorism. The Majority would also note that 'terrorism' has never been singly defined under international law [...] the international community has followed a thematic approach to the characterization of international terrorism, with subject-specific conventions [...] The prohibition of terror against the civilian population in times of war, which (as discussed below) is given expression in Geneva Convention IV and the Additional Protocols, is another example of the thematic, subject-specific, approach to 'terrorism' [...] the Majority has not considered it necessary to enter into discussion of 'political' terrorist violence and of attempts to regulate it through international conventions [...]", Prosecutor v. Stanislav Galić, IT-98-29-T, Trial Chamber, Judgement, 5 December 2003, notas al pie de los paras. 87 y 133 .

25 Artículo $3^{\circ}$ del Estatuto del TPIY. Violaciones de las leyes o prácticas de guerra: "El Tribunal Internacional tiene competencia para perseguir a las personas que cometan violaciones de las leyes o prácticas de guerra [...]".

26 "[...] For a crime to be adjudicated under article $3^{\circ}$ of the Statute (violation of the laws and customs of the war) the Trial Chamber must determine that a state of armed conflict existed at the time the crime was committed and that the crime was 'closely related' to the armed conflict [...]", Prosecutor v. Stanislav Galic, IT-98-29-T, Trial Chamber, Judgement, 5 December 2003, paras. 9 y 10. 
la víctima; 4) la violación de la norma debe implicar responsabilidad penal individual para su autor y así debe desprenderse de normas de Derecho Internacional Consuetudinario o Convencional ${ }^{27}$.

En el caso, la primera condición se consideró cumplida, toda vez que la conducta de aterrorizar civiles en el contexto de conflictos armados se encuentra prohibida por el Derecho Internacional Humanitario, con base en el principio general de protección que goza la población civil en esos contextos, el cual, además, ha sido considerado costumbre internacional por la jurisprudencia del TPIY ${ }^{28}$. La conducta se encuentra prohibida en la segunda parte del segundo subparágrafo del artículo 51 del Protocolo I Adicional a los Convenios de Ginebra (disposición idéntica al subparágrafo 2 del artículo 13 del Protocolo II), que establece: “[...] No serán objeto de ataque la población civil como tal ni las personas civiles. Quedan prohibidos los actos o amenazas de violencia cuya finalidad principal sea aterrorizar a la población civil [...]"29.

La segunda condición generó disidencia en primera instancia y discrepancias entre la postura mayoritaria de primera y segunda instancia.

Según la mayoría del tribunal de juicio, la vigencia de la norma infringida (prohibición de terrorismo) al momento de los hechos y su aplicación al caso tenía base convencional y se fundaba en el Acuerdo del 22 de mayo de 1992, firmado por las partes involucradas en este conflicto armado, el cual no sólo remitía al artículo 51 del Protocolo I Adicional, sino que lo había incluido expresamente en su texto ${ }^{30}$. Por ello, el tribunal entendió que no era necesario pronunciarse sobre la aplicación al conflicto de Protocolos Adicionales a los Convenios de Ginebra ${ }^{31}$, ni tampoco sobre si la regla de

27 Prosecutor v. Duško Tadić, IT-94-1, Trial Chamber, Decision on the Defense Motion for Interlocutory Appeal on Jurisdiction, 10 August 1995, para 94. Prosecutor v. Stanislav Galić, IT-98-29-T, Trial Chamber, Judgement, 5 December 2003, paras. 9/12.

28 Prosecutor v. Stanislav Galić, IT-98-29-T, Trial Chamber, Judgement, 5 December 2003, para. 19.

29 Prosecutor v. Stanislav Galić, IT-98-29-T, Trial Chamber, Judgement, 5 December 2003, paras. 95/96.

30 "[...] the warring parties entered into several agreements under the auspices of the ICRC. The first of these was the 22 May Agreement, by which the parties undertook to protect the civilian population from the effects of hostilities and to respect the principle prohibiting attacks against the civilian population [...] they agreed to bring into force, inter alia, articles 35 to 42 and 48 to 58 of Additional Protocol I [...] by virtue of the 22 May 1992 Agreement the parties to the conflict clearly agreed to abide by the relevant provisions of Additional Protocol I protecting civilians from hostilities. Therefore, article 51 [...] undoubtedly applied as conventional law between the parties to the conflict [...]", Prosecutor v. Stanislav Galić, IT-98-29-T, Trial Chamber, Judgement, 5 December 2003, paras. 22 y 25, remisión en para. 95 in fine.

31 “[...] The Majority emphasizes that it is not required to pronounce on whether the rule 
Derecho Humanitario infringida tenía naturaleza consuetudinaria, dado que alcanzaba con reconocer su aplicación al caso derivada de instrumentos convencionales vinculantes a las partes, vigentes al momento del hecho y no derogados por normas imperativas, conforme lo exigía también el precedente "Tadic" para fundar la jurisdicción del tribunal en normas de Derecho Convencional $^{32}$.

Sobre esto último, se concluyó que: 1) el Acuerdo del 22 de mayo de 1992 -que recogía al art. 51 del Protocolo I- estaba vigente y era vinculante para las partes en conflicto, circunstancia que impedía que una imputación con base en su letra conculcara el principio general de nullum crimen sine lege (cfr. art. 15 del Pacto Internacional de los Derechos Civiles y Políticos); y 2) las disposiciones del acuerdo que prohibían el recurso al terrorismo no sólo no estaban derogadas ni contradecían, sino que reafirmaban normas imperativas de Derecho Internacional; en el caso, aquellas que protegen a la población civil en contexto de conflictos armados ${ }^{33}$.

Por su parte, en su opinión disidente, el juez Nieto-Navia consideró que para respetar el principio nullum crimen sine lege, el tribunal procuraba que los crímenes sometidos a su jurisdicción estuvieran basados en normas de Derecho Internacional Consuetudinario, descartando que la jurisdicción del tribunal pudiera afirmarse en normas de Derecho Convencional ${ }^{34}$.

$\mathrm{Al}$ respecto y a su turno, la cámara de apelaciones sostuvo que, en tanto los acuerdos cumplieran con los requisitos específicos exigidos, el tribunal podía fundar su jurisdicción en normas de Derecho Convencional; no obstante lo cual, recordó que -históricamente- sus jueces se habían inclinado por asegurar que las conductas sometidas a su juzgamiento estuvieran reprimidas, al tiempo de su comisión, por normas de Derecho Internacional Consuetudinario y en tipos penales suficientemente definidos ${ }^{35}$. En conse-

in question is also customary in nature. As stated above, it belongs to 'treaty law' [...] Whether the crime of terror also has a foundation in customary law is not a question which the Majority is required to answer [...]". Prosecutor v. Stanislav Galić, IT-98-29-T, Trial Chamber, Judgement, 5 December 2003, paras. 95/97 y 138.

32 Prosecutor v. Duško Tadić, IT-94-1, Trial Chamber, Decision on the Defense Motion for Interlocutory Appeal on Jurisdiction, 10 August 1995, para 143. Prosecutor v. Stanislav Galić, Case No. IT-98-29-T, Trial Chamber, Judgement, 5 December 2003, para. 98.

33 "[...] the second paragraph of article 51, read as a whole, intends to say [...] that the prohibition against terror is a specific prohibition within the general prohibition of attack on civilians. The general prohibition is a peremptory norm of customary international law [...] by exemplifying and therefore according with the general norm, the rule against terror neither conflicts with nor derogates from peremptory norms of international law [...]', Prosecutor $v$. Stanislav Galić, IT-98-29-T, Trial Chamber, Judgement, 5 December 2003, paras. 98.

34 Prosecutor v. Stanislav Galić, IT-98-29-T, Trial Chamber, Judgement, 5 December 2003, Separate and partially dissenting opinion of Judge Nieto-Navia, paras. 108/113.

35 "[...] However, while conventional law can form the basis for the International 
cuencia, si bien rechazó la postura de la defensa que aducía que la jurisdicción del tribunal bajo artículo $3^{\circ}$ de su Estatuto sólo podía basarse en Derecho Consuetudinario ${ }^{36}$, concluyó que la prohibición de aterrorizar a la población civil consagrada en los artículos 51 (2) y 13 (2) de los Protocolos I y II, respectivamente, constituía una norma de Derecho Internacional Consuetudinario, al menos, desde el instante en que esas disposiciones habían sido incorporadas a los protocolos (1977) y, por tanto, vigentes al momento de los hechos del caso ${ }^{37}$.

En opinión separada, el juez Shahabuddeen sostuvo que el tribunal nunca había asumido jurisdicción exclusivamente con base en Derecho Convencional porque, en realidad, entendía necesario contar con fundamento en la costumbre internacional ${ }^{38}$.

Así, respecto de la "segunda condición", se destaca que: mientras que ambas cámaras reconocieron que la jurisdicción del tribunal podía fundarse en normas de Derecho Internacional Convencional o Consuetudinario, en el caso, la cámara de juicio se basó en Derecho Convencional y la cámara de apelaciones modificó esa decisión y se afirmó en normas de Derecho Consuetudinario.

Si bien la constatación de la tercera "condición Tadič" no conllevó mayores controversias, la cuarta condición acercó las posturas disidentes de los jueces Nieto-Navia y Schomburg, expresadas en primera y segunda instancia, respectivamente, y alineó las posiciones de las mayorías de las cámaras de juicio y apelaciones.

Tribunal's jurisdiction, provided that the above conditions are met, an analysis of the jurisprudence of the International Tribunal demonstrates that the Judges have consistently endeavoured to satisfy themselves that the crimes charged in the indictments before them were crimes under customary international law at the time of their commission and were sufficiently defined under that body of law [...]”, Prosecutor v. Stanislav Galić, IT-98-29-A, Appeals Chamber, Judgement, 30 November 2006, paras. 82/83.

36 Prosecutor v. Stanislav Galić, IT-98-29-A, Appeals Chamber, Judgement, 30 November 2006, paras. 85/6.

37 Para así decidirlo, la cámara analizó el proceso de adopción de los protocolos adicionales a los Convenios de Ginebra, sus travaux préparatoires y su elevada tasa de ratificación; asimismo, invocó varios instrumentos internacionales que contenían la prohibición y también detalló distintas prácticas de los Estados (declaraciones públicas, manuales militares, etc.). Además, se afirmó: "[...] The Appeals Chamber therefore affirms the finding of the Trial Chamber that the prohibition of terror, as contained in the second sentences of both article 51(2) of Additional Protocol I and article 13(2) of Additional Protocol II, amounts to a specific prohibition within the general (customary) prohibition of attack on civilians [...] clearly belonged to customary international law [...]', Prosecutor v. Stanislav Galić, IT-98-29-A, Appeals Chamber, Judgement, 30 November 2006, paras. 86/88 y 90.

38 Prosecutor v. Stanislav Galić, IT-98-29-A, Appeals Chamber, Judgement, 30 November 2006, Separate Opinion of Judge Shahabuddeen, para. 2. 
A pesar de no haber analizado y/o reconocido la existencia de base consuetudinaria en relación con el crimen de terrorismo como violación a las leyes y prácticas de la guerra, la mayoría de la sala de juicio sostuvo que, de todas formas, le correspondía constatar si en 1992 existía alguna norma, convencional o consuetudinaria, aplicable al caso, que contemplara la atribución de responsabilidad penal individual por violación a la prohibición de terrorismo ${ }^{39}$. $\mathrm{Al}$ respecto, concluyó que aun cuando la violación a la prohibición de terrorismo se imputara como la conculcación al Acuerdo del 22 de mayo de 1992 y no como grave violación al artículo 51 (2) del Protocolo I Adicional a los Convenios de Ginebra, en 1992 cabía atribuir responsabilidad penal individual por serias violaciones a la prohibición de terrorismo prevista en el artículo 51 (2) del Protocolo I, cuando ésta importara graves lesiones o la muerte de civiles ${ }^{40}$.

Con esa conclusión, se entendió garantizada la indemnidad del principio general de nullum crimen sine lege. Se aclaró que lo decidido no implicaba imputar los hechos como graves violaciones a disposiciones del Protocolo I, ni afirmar la jurisdicción del tribunal o pronunciarse sobre otras posibles formas de vulnerar la prohibición de terrorismo (v. gr., amenazas o actos de violencia que no causen muerte o lesiones graves), toda vez que la intervención del tribunal de juicio había quedado circunscripta al supuesto fáctico en estudio ${ }^{41}$.

En la misma línea, la mayoría de la cámara de apelaciones concluyó que el Derecho Consuetudinario autorizaba a atribuir responsabilidad penal individual por violaciones a la prohibición de aterrorizar a la población civil consagradas en los artículos 51 (2) y 13 (2) de los Protocolos Adicionales, al menos, en el período correspondiente a los hechos del caso ${ }^{42}$.

39 Prosecutor v. Stanislav Galić, IT-98-29-T, Trial Chamber, Judgement, 5 December 2003, para. 113.

40 Ver los distintos argumentos, en especial aquel que lo trata como "una especie de los ataques ilegales contra civiles, siempre que importen lesiones graves o muerte [...]", Prosecutor v. Stanislav Galić, IT-98-29-T, Trial Chamber, Judgement, 5 December 2003, paras. 127/129.

41 " [...] the Majority finds that serious violations of the second part of article 51(2), and specifically the violations alleged in this case causing death or injury, entailed individual criminal responsibility in 1992. The Majority expresses no view as to whether the Tribunal also has jurisdiction over other forms of violation of the rule, such as the form consisting only of threats of violence, or the form comprising acts of violence not causing death or injury [...] The Majority finds that the offence as here defined was criminalized in a precise and accessible manner by 1992, and that this was known or should have been known to the Accused. Therefore, there is no affront to the principle of nullum crimen sine lege [...]", Prosecutor $v$. Stanislav Galić, IT-98-29-T, Trial Chamber, Judgement, 5 December 2003, paras. 130 y 132.

42 Para así decidir, según lineamientos del precedente "Tadić", el tribunal analizó la práctica reiterada de los Estados (declaraciones públicas, legislación y judicialización a nivel 
Por sus fundamentos, el juez Meron también concurrió con la mayoría ${ }^{43}$. Por separado, el juez Shahabuddeen coincidió con la mayoría en que existía base consuetudinaria aplicable al caso para fundar la atribución de responsabilidad penal individual por terrorismo, pero aclaró que ello no conllevaba afirmar que existiera opinio juris ni práctica reiterada por parte de los Estados que sustentaran una definición comprensiva y consuetudinaria de terrorismo. Señaló que la comunidad internacional había consensuado sobre una noción básica, pero persistían diferencias significativas y que no convenía pronunciarse sobre una norma consuetudinaria en desarrollo $0^{44}$.

Las coincidentes mayorías en ambas instancias confrontaron con las disidencias de los jueces Nieto-Navia y Schomburg, quienes, cada cual a su turno, entendieron que el tribunal no tenía jurisdicción para juzgar la conducta de aterrorizar a la población civil durante un conflicto armado, porque no se había logrado demostrar que la misma suscitara responsabilidad penal individual en normas de Derecho Internacional Consuetudinario. Si bien la prohibición de infligir terror a los civiles ya integraba la costumbre internacional, el análisis de la práctica y opinio iuris de los Estados revelaba que la conducta no se encontraba criminalizada en ese entonces, por lo que la sentencia condenatoria, en los términos en los que había sido dictada, vulneraba el principio fundamental de nullum crimen sine lege ${ }^{45}$. Schomburg añadió que, consecuentemente, correspondía anular la calificación y encuadrar la conducta atribuida a Stanislav Galić en la figura de ataques

nacional, manuales militares, etc.). Prosecutor v. Stanislav Galić, IT-98-29-A, Appeals Chamber, Judgement, 30 November 2006, para. 86, 92 y 98. Cfr. Prosecutor v. Duško Tadić, IT-94-1, Trial Chamber, Decision on the Defense Motion for Interlocutory Appeal on Jurisdiction, 10 August 1995, para 128.

43 Prosecutor v. Stanislav Galić, IT-98-29-A, Appeals Chamber, Judgement, 30 November 2006, Separate and Partially Dissenting Opinion of Judge Meron, para. 2.

44 "I agree with the view that terror as charged is a crime known to customary international law [...] in taking up that position [...] not suggesting that a comprehensive definition of terror is known to customary international law; the Appeals Chamber is really speaking of a core concept [...] The developing state of the law gives reason for caution [...] I am satisfied that a serious violation of 'the laws or customs of war' within the meaning of article $3^{\circ}$ of the Statute, namely, by resorting to the core of terror, gives rise to such responsibility. I am also satisfied that this responsibility existed at the time of the alleged acts of the appellant [...]", Prosecutor v. Stanislav Galić, IT-98-29-A, Appeals Chamber, Judgement, 30 November 2006, Separate Opinion of Judge Shahabuddeen, paras. 3/5.

45 Prosecutor v. Stanislav Galić, IT-98-29-T, Trial Chamber, Judgement, 5 December 2003, Separate and partially dissenting opinion of Judge Nieto-Navia, paras. 108/113. Prosecutor v. Stanislav Galić, IT-98-29-A, Appeals Chamber, Judgement, 30 November 2006, Separate and partially dissenting opinion of Judge Schomburg. 
ilegales contra civiles, valorando la finalidad de aterrorizar a la población como circunstancia agravante de la pena ${ }^{46}$.

Recapitulando, por mayoría y en ambas instancias, el tribunal consideró cumplidas las cuatro "condiciones de Tadić" y, consecuentemente, asumió la jurisdicción sobre los hechos y se pronunció. Como se advierte en estas líneas, el análisis de los argumentos, mayoritarios y disidentes, en torno a la constatación de estas "condiciones", examina aspectos significativos en materia de caracterización y criminalización del terrorismo en contextos de conflicto armado.

\section{2.b. La caracterización del crimen de terrorismo}

La mayoría del tribunal de juicio definió al terrorismo como un crimen de guerra autónomo, integrado por elementos comunes a las restantes violaciones a las leyes o usos de la guerra admitidas por el Estatuto ${ }^{47}$, así como por los siguientes elementos objetivos y subjetivos propios: 1) actos de violencia dirigidos contra la población civil o contra individuos civiles que no participan de las hostilidades, y que causan la muerte o graves lesiones al cuerpo o a la salud de la población civil; 2) que en forma deliberada, el autor convierta a la población civil o a individuos civiles que no participan de las hostilidades, en el objetivo de esos actos de violencia; 3) que las acciones previamente descriptas hayan sido cometidas con el principal propósito de propagar terror en la población civil ${ }^{48}$.

46 "The International Tribunal is required to adhere strictly to the principle of nullum crimen sine lege praevia and must ascertain that a crime was 'beyond any doubt part of customary international law'. It would be detrimental not only to the Tribunal but also to the future development of international criminal law and international criminal jurisdiction if our jurisprudence gave the appearance of inventing crimes -hus highly politicizing its functions- where the conduct in question was not without any doubt penalized at the time when it took place", Prosecutor v. Stanislav Galić, IT-98-29-A, Appeals Chamber, Judgement, 30 November 2006, Separate and Partially Dissenting Opinion of Judge Schomburg, paras. 14/21.

47 La existencia de un conflicto armado al momento de los hechos y la vinculación entre el crimen y ese conflicto armado.

48 "133. In conclusion, the crime of terror against the civilian population in the form charged in the Indictment is constituted of the elements common to offences falling under article $3^{\circ}$ of the Statute, as well as of the following specific elements: 1. Acts of violence directed against the civilian population or individual civilians not taking direct part in hostilities causing death or serious injury to body or health within the civilian population. 2. The offender willfully made the civilian population or individual civilians not taking direct part in hostilities the object of those acts of violence. 3. The above offence was committed with the primary purpose of spreading terror among the civilian population”. Prosecutor v. Stanislav Galić, IT-98-29-T, Trial Chamber, Judgement, 5 December 2003, para. 133. 
De acuerdo con esta definición, el crimen de terrorismo se configura con los elementos objetivos y subjetivos del crimen de guerra que tipifica los ataques ilegales contra civiles con resultado muerte o lesión grave al cuerpo o a la salud (apartados 1 y 2 del párrafo anterior), pero con un elemento subjetivo adicional y propio, que consiste en realizar el tipo objetivo con el principal propósito de aterrorizar a la población civil (apartado 3). Ambos crímenes tutelan la protección de la población civil en el marco de conflictos armados, que se deriva del principio de distinción. En línea con ello, el artículo 51 (2) del Protocolo I Adicional a los Convenios de Ginebra ha sido considerado un instrumento que ha complementado y reforzado, con mayor especificidad, el Derecho Consuetudinario preexistente. En el caso, por el principio de especialidad, los hechos quedaron calificados en la figura de terrorismo y no como ataques contra civiles ${ }^{49}$.

En lo concerniente a la definición general de la noción de "terror", en el juicio se aceptó la noción aportada por la fiscalía, que lo calificaba como "miedo extremo" ${ }^{50}$, y ello fue ratificado en segunda instancia ${ }^{51}$.

\section{2.b.1. Actus reus. Aspecto objetivo del tipo penal de terrorismo}

En primer lugar, vale señalar que la conducta prohibida en el artículo 51 (2) del Protocolo I consiste en ataques dirigidos contra la población civil o individuos civiles que no se encuentran participando de las hostilidades. Por ataque, el citado protocolo entiende a los actos de violencia-ofensivos o defensivos- (art. 49), mientras que la jurisprudencia del tribunal lo define como un "curso de acción que involucra la comisión de actos de violencia"52. En segundo lugar y en lo atinente a los sujetos pasivos, se trata de individuos "civiles" o de la "población civil" en general, es decir, aquellas personas que no pertenezcan a las fuerzas armadas o grupos militares organizados que respondan a las partes en conflicto ${ }^{53}$. Finalmente y en lo concerniente al resultado como elemento objetivo del tipo, la mayoría concluyó que la figura no contempla el resultado de haber aterrorizado civiles en forma efectiva,

49 Prosecutor v. Stanislav Galić, IT-98-29-T, Trial Chamber, Judgement, 5 December 2003, paras. $42,46,56,62,159 / 162$.

50 Prosecutor v. Stanislav Galić, IT-98-29-T, Trial Chamber, Judgement, 5 December 2003, para. 137.

51 Prosecutor v. Stanislav Galić, IT-98-29-A, Appeals Chamber, Judgement, 30 November 2006, nota al pie 320 en para. 103.

52 Prosecutor v. Stanislav Galić, IT-98-29-T, Trial Chamber, Judgement, 5 December 2003, paras. 52/53.

53 Prosecutor v. Stanislav Galić, IT-98-29-T, Trial Chamber, Judgement, 5 December 2003, paras. 47 y 137. 
por lo que no es necesario probar ese extremo ni su conexión causal con los actos de violencia que conforman la conducta prohibida ${ }^{54}$. Esto fue ratificado en segunda instancia ${ }^{55}$.

Por entender que excedía el caso, el tribunal de juicio omitió pronunciarse sobre si el crimen de terrorismo se configuraba en supuestos en que la conducta consistiera en amenazas de violencia (no actos) o en actos de violencia que no causaran muerte o lesiones graves. A su turno, la cámara de apelaciones entendió que correspondía incluir a las amenazas de violencia entre las conductas típicas ${ }^{56}$. Aclaró que los "actos o amenazas de violencia" incluyen a los "ataques y amenazas de ataques" y que, si bien pueden ser directos, también pueden variar su naturaleza y ser indiscriminados o desproporcionados, siempre y cuando fueran cometidos con el propósito principal de propagar el terror ${ }^{57}$.

\section{2.b.2. Mens rea. Aspecto subjetivo del tipo penal de terrorismo}

$\mathrm{Al}$ igual que la figura criminal del ataque ilegal contra civiles que resulte en muerte o lesión grave al cuerpo o a la salud, el crimen de terrorismo exige que, "en forma deliberada", el autor convierta a la población civil o a individuos civiles que no participan de las hostilidades, en el objetivo de esos actos de violencia (punto 2 de ambas definiciones). En otras palabras, exige un dolo general, un accionar consciente e intencionado respecto de los actos violentos capaces de configurar el tipo objetivo (homicidios, torturas, malos tratos, ataques contra civiles, etc. ${ }^{58}$.

No obstante, en forma concomitante, esta figura requiere que el tipo objetivo sea realizado con una intencionalidad específica, en concreto, con

54 Prosecutor v. Stanislav Galić, IT-98-29-T, Trial Chamber, Judgement, 5 December 2003 , paras. 65 y $134,151$.

55 "[...] actual terrorization of the civilian populations is not an element of the crime [...]", Prosecutor v. Stanislav Galić, IT-98-29-A, Appeals Chamber, Judgement, 30 November 2006, paras. 103/104.

56 Prosecutor v. Stanislav Galić, IT-98-29-A, Appeals Chamber, Judgement, 30 November 2006, para. 101.

57 “'...] Article 49(1) of Additional Protocol I defines 'attacks' as 'acts of violence'. Accordingly, the crime of acts or threats of violence [...] can comprise attacks or threats of attacks against the civilian population [...] [it] shall not however be limited to direct attacks against civilians or threats thereof but may include indiscriminate or disproportionate attacks or threats thereof [...]", Prosecutor v. Stanislav Galić, IT-98-29-A, Appeals Chamber, Judgement, 30 November 2006, para. 102.

58 ' [...] the notion of 'wilfully' incorporates the concept of recklessness, whilst excluding mere negligence. The perpetrator who recklessly attacks civilians acts 'wilfully' [...]", Prosecutor v. Stanislav Galić, IT-98-29-T, Trial Chamber, Judgement, 5 December 2003, para. 54. 
el propósito principal de propagar terror en la población civil. Este dolo especial que requiere el crimen de terrorismo constituye, sin más, su característica distintiva, aquella que lo diferencia del resto de las figuras y, en particular, de los ataques ilegales contra la población civil con resultado muerte o lesión grave.

Desde la perspectiva de la dogmática penal, el dolo general previsto para la realización de los actos violentos admite dolo eventual y excluye las conductas culposas, siempre respecto de los ataques o amenazas dirigidos contra civiles. En cambio, el dolo especial exigido para considerar configurado el crimen de terrorismo, solo admite dolo directo, circunstancia que excluye las acciones ejecutadas con dolo eventual o culpa. Esto último importa que no alcance con demostrar que el autor debió representarse la posibilidad de que sus acciones ilegales podían aterrorizar a civiles o, incluso, que ese resultado se produzca como consecuencia colateral y no intencionada de los actos ilegales. En suma, el crimen de terrorismo exige que su autor ejecute la acción típica con la intención primaria de aterrorizar a la población civil $^{59}$.

La cámara de apelaciones añadió que, en el aspecto subjetivo, la intención de propagar terror podía coexistir con otras motivaciones, siempre y cuando aquel constituyera el propósito principal de la acción típica. Asimismo, aclaró que esa intencionalidad podía inferirse de circunstancias de los actos o amenazas, tales como su naturaleza, modalidad, oportunidad, duración, etcétera ${ }^{60}$.

\section{Jurisprudencia posterior en el TPIY: Después de "Galić"}

La importancia del caso "Galić" no solamente se avizora de cara a las cuestiones que logró sintetizar, en forma superadora, respecto de sus antecedentes, sino que, especialmente, se revela en los casos que lo sucedieron, los cuales permiten conocer el modo en que su doctrina se derramó en la jurisprudencia del tribunal y, no menos importante, la forma en que los

59 “[...] 'Primary purpose' signifies the mens rea of the crime of terror. It is to be understood as excluding dolus eventualis or recklessness from the intentional state specific to terror [...] The crime of terror is a specific-intent crime [...] excludes terror which was not intended by a belligerent and terror that is merely an incidental effect of acts of warfare which have another primary object and are in all other respects lawful [...]", Prosecutor $v$. Stanislav Galić, IT-98-29-T, Trial Chamber, Judgement, 5 December 2003, paras. 136 y 101.

60 Prosecutor v. Stanislav Galić, IT-98-29-A, Appeals Chamber, Judgement, 30 November 2006, para. 104. 
casos posteriores moldearon la doctrina original hasta derivar en su última versión.

En línea con ello, corresponde iniciar la revisión de este segundo conjunto de casos, con las sentencias de sendas instancias dictadas en Prosecutor v. Dragomir Milošević, quien sucedió a Stanislav Galić en el comando del asedio a Sarajevo y fue consecuentemente condenado, entre otros crímenes, por terrorismo, a la pena de treinta y tres años de prisión, sanción que fue reducida a veintinueve años en segunda instancia ${ }^{61}$.

Entre los aportes de la sentencia de juicio en "Milošević" a la doctrina del precedente "Galić", cabe mencionar: 1) se consolidó la definición que caracterizó el crimen de terrorismo conforme las modificaciones introducidas por la sentencia de apelación en "Galić"; 2) se convalidó que podían existir motivaciones concomitantes al propósito principal exigido, pero no era necesaria su comprobación; 3) se recordó que la figura exigía el resultado muerte o grave lesión al cuerpo o a la salud, pero no el hecho de haber infligido terror a la población civil en forma efectiva, a lo cual se agregó que, no obstante lo dicho, la constatación de semejante resultado podía contribuir para la demostración de la intención de aterrorizar típicamente requerida; 4) se brindaron distintos criterios a los fines de favorecer la comprobación del dolo especial; 5) se precisó que para que la conducta sea penalmente relevante, debe constatarse -en el caso concreto- la intención de infundir miedo por sobre el temor basal naturalmente derivado de la situación de enfrentamiento armado; etc. En línea con "Galić", el tribunal de juicio concluyó: "El crimen de terrorismo, en consecuencia, constituye una forma 'agravada', más severa, de ataque ilegal a civiles"62.

Se criticó la escasa profundización del precedente respecto a la noción de "terror" y, si bien reconoció una creciente tendencia a diferenciar el terrorismo en tiempos de paz y de guerra, señaló que -en cualquier caso- los ataques contra la población civil se encuentran prohibidos en ambos casos por el Derecho Internacional ${ }^{63}$.

61 "[...] The Appeals Chamber held that the crime of terror was both prohibited and criminalized under customary international law. The crime falls under article $3^{\circ}$ of the Statute as a violation of the laws or customs of war and the Tribunal has jurisdiction over the crime [...]", Prosecutor v. Dragomir Milošević, IT-98-29/1-T, Trial Chamber, Judgement, 12 December 2007, para. 874. Prosecutor v. Dragomir Milošević, IT-98-29/1-A, Appeals Chamber, Judgement, 12 November 2009.

62 "[...] The prohibition of spreading terror among a civilian population must therefore always be distinguished from the effects that acts of legitimate warfare can have on a civilian population [...]', Prosecutor v. Dragomir Milošević, IT-98-29/1-T, Trial Chamber, Judgement, 12 December 2007, paras. 875, 879/882, 888.

63 Prosecutor v. Dragomir Milošević, IT-98-29/1-T, Trial Chamber, Judgement, 12 December 2007, paras. 883 y 887 . 
A su turno, la cámara de apelaciones - por mayoría- aseveró que la sentencia de juicio había incurrido en error de derecho al exigir la causación del resultado muerte o lesión grave al cuerpo o a la salud, como elemento del crimen de terrorismo. A la luz de este enfoque, esas acciones (causar muerte o lesionar gravemente) representan modos posibles de comisión del crimen, pero no deben ser considerados elementos del tipo que, por otra parte, sí exige que las víctimas sufran graves consecuencias como resultado de los actos o amenazas de violencia, que incluyen, pero no se circunscriben, a la muerte o a lesiones graves ${ }^{64}$.

Por su parte, el juez Liu Daqun se alineó con las disidencias de NietoNavia y Schomburg en "Galić", en tanto no reconoció norma consuetudinaria vigente al momento de los hechos que criminalizara la prohibición de aterrorizar civiles en conflictos armados y concluyó que los hechos debían encuadrarse en la figura de ataques ilegales contra civiles y que el terror debía ser valorado como agravante de ese crimen y no un crimen en sí mismo. A su juicio, la eliminación del requisito de producir muerte o lesiones graves desatendía el cumplimiento de la tercera "condición Tadić" y que, por lo tanto, la mayoría no había identificado adecuadamente los elementos constitutivos del crimen de terrorismo, generando una indefinición inaceptable en Derecho Penal ${ }^{65}$.

Ahora bien, retomando la cronología de casos, corresponde seguir con Prosecutor v. Milan Martić, en el que la cámara de apelaciones del tribunal aludió a la caracterización del crimen de terrorismo realizada en el precedente "Galić", pero únicamente a los fines de diferenciarlo del crimen de ataque contra civiles ${ }^{66}$.

En Prosecutor v. Vujadin Popović et al., se condenó a varios acusados por múltiples hechos y en la descripción de la plataforma fáctica se advierten numerosas alusiones al terror infundido a las víctimas. Las diferentes acciones que significaron aterrorizar a la población civil bosnio-musulmana fueron calificadas como actos persecutorios constitutivos de crímenes contra la humanidad, tal como ocurriera en los primeros precedentes analizados (cfr. "Krstić" y "Nikolić", etc.) ${ }^{67}$.

64 Prosecutor v. Dragomir Milošević, IT-98-29/1-A, Appeals Chamber, Judgement, 12 November 2009, para. 33.

65 Prosecutor v. Dragomir Milošević, IT-98-29/1-A, Appeals Chamber, Judgement, 12 November 2009, Partly Dissenting Opinion of Judge Liu Daqun, paras. 13/14 y 17/20.

66 Prosecutor v. Milan Martić, IT-95-11-A, Appeals Chamber, Judgement, 8 October 2008, para. 259/260.

67 "The Trial Chamber therefore concludes that the underlying acts of [...] terrorizing civilians [...] constitute persecution as defined in article $5^{\circ}$ of the Statute.. committed in the context of a widespread and systematic attack and thus constitute crimes against humanity", Prosecutor v. Vujadin Popović et al., IT-05-88-T, Trial Chamber, Judgement, 10 June 2010, 
En especial, el tribunal de juicio encuadró en los requisitos propios del crimen de terrorismo a ciertos actos de persecución (que involucraban aterrorizar a la población civil) y concluyó que configuraban crímenes de lesa humanidad $^{68}$. Este particular abordaje recibió el reproche del superior, que aclaró que la persecución como crimen contra la humanidad no requiere que los actos persecutorios configuren -a su vez- crímenes internacionales $\mathrm{y}$, por tanto, no resultaba necesario demostrar que las conductas persecutorias cometidas con intención discriminatoria configuraban, además, el crimen de terrorismo $0^{69}$. No obstante lo cual, ambas instancias coincidieron en reconocer que la comprobación de esos extremos revestía importancia a los fines de valorar la gravedad de las conductas atribuidas ${ }^{70}$.

En Prosecutor v. Zdravko Tolomir, al igual que en "Popović", la cámara de juicio argumentó que la exposición a las acciones y amenazas terroristas conculcaban el derecho fundamental a la seguridad individual, consagrado en el Derecho Internacional de los Derechos Humanos y que, por lo tanto, la acción de aterrorizar civiles revestía la suficiente gravedad como para configurar actos de persecución, constitutivos de crímenes contra la humanidad $^{71}$.

La relevancia política del imputado atravesó el caso Prosecutor $v$. $R a$ dovan Karadžić. Entre otras imputaciones, se lo condenó por persecución, en tanto crimen de lesa humanidad y por terrorismo, en línea con los precedentes "Galić" y su progenie ${ }^{72}$. El tribunal ratificó al crimen de terrorismo como una violación a las leyes y usos de la guerra, y se consolidó la

paras. 777, 834, 844/45, 963, 970, 996/999, 1004, entre muchos otros. Prosecutor v. Vujadin Popović et al., IT-05-88-A, Appeals Chamber, Judgement, 30 January 2015.

68 Prosecutor v. Vujadin Popović et al., IT-05-88-T, Trial Chamber, Judgement, 10 June 2010, paras. 979/981.

69 Prosecutor v. Vujadin Popović et al., IT-05-88-A, Appeals Chamber, Judgement, 30 January 2015, paras. 737/738 y 763 .

70 "[...] Accordingly, the terrorization of civilians is of sufficient gravity to amount to persecution", Prosecutor v. Vujadin Popović et al., IT-05-88-T, Trial Chamber, Judgement, 10 June 2010, para. 981. "[...] the primary purpose of the underlying acts may have been relevant to the gravity of the acts in question [...]", Prosecutor v. Vujadin Popovic et al., IT-05-88-A, Appeals Chamber, Judgement, 30 January 2015, para 763.

71 Prosecutor v. Vujadin Popović et al., IT-05-88-T, Trial Chamber, Judgement, 10 June 2010, para. 981. "[...] Exposure to such acts or threats constitutes a violation of an individual's fundamental right to security of person, which is enshrined in various international and regional human rights treaties. Thus, the terrorization of civilians is an act of sufficient gravity to constitute persecutions [...]", Prosecutor v. Zdravko Tolomir, IT-05-88/2-T, Trial Chamber, Judgement, 12 December 2012, paras. 855/857, 868, 1188, etc. Prosecutor v. Zdravko Tolomir, IT-05-88/2-A, Appeals Chamber, Judgement, 8 April 2015.

72 Prosecutor v. Radovan Karadžić, IT-95-5/18-T, Trial Chamber, Judgement, 24 March 2016. 
siguiente definición: 1) actos o amenazas de violencia dirigidos contra la población civil o individuos civiles que no participan de las hostilidades; 2) que en forma deliberada, el autor convierta a la población civil o a individuos civiles que no participan de las hostilidades, en el objetivo de esos actos o amenazas de violencia; 3) que las acciones previamente descriptas hayan sido cometidas con el principal propósito de propagar terror en la población civil ${ }^{73}$.

En Prosecutor v. Jadranko Prlić et al., se condenó -entre otros crímenes- por terrorismo como crimen de guerra, de conformidad con "Galić" y su progenie ${ }^{74}$. Por mayoría, la cámara de apelaciones confirmó las condenas impuestas y, en una nueva disidencia, el juez Liu Daqun reprodujo todos los fundamentos de su opinión en "Dragomir Milošević"75.

Finalmente, en Prosecutor v. Ratko Mladić se dictó condena a prisión perpetua, entre otros, por el crimen de terrorismo (por los hechos ocurridos en Sarajevo), en línea con la jurisprudencia del tribunal ${ }^{76}$.

De los antecedentes someramente revisados en este apartado, cabe concluir que, por un lado, el tribunal continuó reprimiendo conductas con contenido terrorista, encuadrándolas en los actos subyacentes de otros crímenes comprendidos en su jurisdicción, tal el caso de los crímenes contra la humanidad. Sin embargo, también se recogió y aplicó la doctrina elaborada por el tribunal en materia de terrorismo, reforzándola, pero no sin modificar algunos de sus aspectos sustantivos.

En términos de conceptualización del crimen, la figura penal delineada por el tribunal de juicio sufrió dos alteraciones significativas en el actus reus: 1) la cámara de apelaciones, en "Galić", incorporó las amenazas de violencia como conducta típica, y 2) la cámara de apelaciones, en "Dragomir Milošević", eliminó el requisito de causación de muerte o lesiones graves en el cuerpo o en la salud de las víctimas civiles. Esta versión "revisada" del

73 "[...] 459. The following elements need to be established before the Chamber can enter a conviction for terror: (a) acts or threats of violence directed against the civilian population or individual civilians not taking direct part in hostilities; (b) the perpetrator wilfully made the civilian population or individual civilians not taking direct part in hostilities the object of those acts of violence; (c) the above was committed with the primary purpose of spreading terror among the civilian population [...]", Prosecutor v. Radovan Karadžić, IT-95-5/18-T, Trial Chamber, Judgement, 24 March 2016, paras. 438/444 y 458/466, 4629/4634.

74 Prosecutor v. Jadranko Prlić et al., IT-04-74-T, Trial Chamber, Judgement, 29 May 2013, Vol I, paras. 26, 140, 194/197; Vol. III, paras. 1689, 1692, etc.

75 Prosecutor v. Jadranko Prlić et al, IT-04-74-A, Appeals Chamber, Judgement, 29 Nov. 2017, Partially Dissenting, Dissenting Opinions and Declaration of Judge Liu Daqun, Vol. III, paras. $8 / 10$.

76 Prosecutor v Ratko Mladić, IT-09-92-T, Trial Chamber, Judgement, 22 November 2017, Parte IV, paras. 4741, 4808, 4892/3, 4921 y 5190. 
tipo penal de terrorismo en contexto de conflictos armados fue aplicada y convalidada, más recientemente, en "Karadžić".

\section{Reflexiones finales}

El presente artículo compila en forma selectiva y secuenciada una serie de aportes jurisprudenciales elaborados por las cámaras del TPIY con relevancia en la caracterización y criminalización del terrorismo en contextos de conflicto armado.

Como se desprende de estas páginas, el paradigmático caso Prosecutor $v$. Stanislav Galić constituyó una bisagra en la jurisprudencia internacional en materia de terrorismo, que excedió la casuística del TPIY, por haber sido la primera vez que un tribunal internacional dictaba sentencia condenatoria por terrorismo como crimen de guerra autónomo, para lo cual -al no encontrarse expresamente previsto en su Estatuto- debió fundar su jurisdicción y caracterizar los aspectos objetivos y subjetivos del tipo penal.

El trabajo se detiene especialmente en el contenido de esa decisión (en ambas instancias) y en el análisis del impacto que la misma representó al interior del propio tribunal internacional que la había adoptado.

En ese sentido, la revisión de la jurisprudencia anterior al caso permite revelar que, desde un comienzo, el tribunal había reconocido y documentado acciones o prácticas vinculadas al terrorismo y, en sus pronunciamientos, había demostrado vocación por asegurar su criminalización. Esa jurisprudencia temprana evidencia que, si bien el terrorismo no fue reprimido mediante la aplicación de un tipo penal específico, el contenido terrorista de las conductas juzgadas no quedó impune, sino que fue penalmente reprochado, ya sea como agravante o como elemento configurativo de alguno de los crímenes expresamente previstos en la competencia del tribunal.

Por su parte, el estudio de la jurisprudencia posterior al caso corroboró que el citado precedente fue aplicado y -a la vez- modificado por sucesivos fallos que, con ajustes y precisiones, contribuyeron sensiblemente a la construcción de una más acabada caracterización del crimen de terrorismo en contexto de conflictos armados.

De acuerdo -justamente- con la doctrina que emana el caso "Galić" y su progenie (fundamentalmente, "Dragomir Milošević" y "Karadžić), el terrorismo como crimen de guerra importa -en su última versión- la comisión de actos o amenazas de violencia dirigidos contra la población civil o individuos civiles que no participan de las hostilidades, siempre y cuando su autor, en forma deliberada, convierta en objetivo de esos actos o amenazas a la población civil o a individuos civiles que no participan de las hostilidades, siendo imprescindible que las acciones previamente descriptas 
sean cometidas con el principal propósito de propagar terror en la población civil.

De todos sus elementos objetivos y subjetivos, el crimen de terrorismo en contextos de conflicto armado elaborado por el TPIY presenta su rasgo distintivo en el aspecto subjetivo del tipo (mens rea), el cual radica en el dolo especial que debe motorizar la realización de la conducta típica; en otras palabras, que los actos o amenazas de violencia sean cometidos con el propósito principal de propagar terror a la población civil.

El abordaje jurisprudencial cronológico y secuenciado del presente análisis ha permitido identificar los distintos aportes que intervinieron en el proceso de construcción colectiva de esta definición pretoriana de terrorismo.

El TPIY se limitó a conceptualizar al terrorismo del régimen legal desarrollado para contextos de conflicto armado, expresado en el IV Convenio de Ginebra y ambos Protocolos Adicionales ${ }^{77}$. Conforme queda aquí evidenciado, el mandato estatuido para el tribunal por el Consejo de Seguridad de Naciones Unidas se tradujo, conceptualmente, en su definición de terrorismo. Dicho de otro modo, el concepto acuñado se encuentra indefectiblemente atravesado por distintos aspectos que hacen a la jurisdicción del tribunal, que ha circunscripto al terrorismo, en términos materiales, al contexto de conflicto armado y, en términos témporo-espaciales, a la constatación de la existencia de la prohibición y criminalización del terrorismo en normas de Derecho Internacional vigentes y aplicables al caso, al momento y en el lugar de los hechos, es decir, a partir de 1991 en el territorio de la Antigua Yugoslavia.

En este punto, resulta sustancial reconocer aquello que Antonio Cassese describió como la multifacética noción criminal de terrorismo en el Derecho Internacional ${ }^{78}$. La jurisprudencia del TPIY en materia de terrorismo constituye un valioso aporte en la definición del terrorismo como crimen de guerra autónomo, como crimen internacional en contextos de conflicto armado. Sin embargo, no es la única definición jurisprudencial sobre ese tipo de terrorismo ${ }^{79}$, no aborda ni zanja todos los problemas conceptuales que

$77 \mathrm{Al}$ respecto, vale aclarar que ni las Convenciones de Ginebra ni los Protocolos Adicionales definen expresamente al terrorismo. Ver: Jodoin, S. (2007). "Terrorism as a War Crime". International criminal Law Review 7, 77-115.

78 Cassese, A. (2006). "The Multifaceted Criminal Notion of Terrorism in International Law”. Journal of International Criminal Justice 4(5): 933-958.

79 El TESL, cuyo estatuto incluía expresamente al terrorismo en su jurisdicción, acuñó una definición propia, que recogió expresamente elementos de la definición elaborada por la jurisprudencia del TPIY, pero se le reconocen rasgos propios y diferenciados (v. gr., los supuestos de actos cometidos contra propiedad civil, la noción de persona protegida, etc.). Para profundizar: Swinnen, J. “Sobre los 'actos de terrorismo' en el Derecho Internacional Humanitario. 
ciertos supuestos representan para la definición del terrorismo en tiempos de guerra ${ }^{80}$ y nada dice sobre los esquemas normativos creados para combatir al terrorismo internacional en tiempos de paz ${ }^{81}$.

Estas precisiones sobre los alcances del aporte que significa la jurisprudencia del Tribunal Penal Internacional para la Antigua Yugoslavia en materia de terrorismo, no le restan importancia, sino que -por el contrario- resaltan el espacio que la misma ocupa en el marco de los persistentes esfuerzos encaminados a consensuar universalmente definiciones para criminalizar este particular modo de ejercer violencia.

\section{Bibliografía}

\section{Jurisprudencia del TPIY}

\section{Prosecutor v. Duško Tadić a/k/a / "DULE"}

IT-94-1, Trial Chamber, Decision on the Defense Motion for Interlocutory. Appeal on Jurisdiction, 10 August 1995.

IT-94-1-T, Trial Chamber, Opinion and Judgment, 7 May 1997.

Prosecutor v. Tihomir Blaskic

IT-95-14-T, Trial Chamber, Judgment, 3 March 2000.

IT-95-14-T, Appeals Chamber, Judgment, 29 July 2004.

Prosecutor v. Dario Kordi et Mario Cerkez

IT-95-14/2-T, Trial Chamber, Judgment, 26 February 2001.

IT-95-14/2-T, Appeals Chamber, Judgment, 17 December 2004.

Una mirada desde los Convenios y Protocolos de Ginebra”. Prudentia Iuris, No 86; Paredi, L., ob. cit., 9-10, 13-14 y 16. Prosecutor v. Moinina Fofana and Allieu Kondewa, SCSL-04-14-T, Trial Chamber, Judgement, 2 August 2007. Prosecutor v. Alex Tamba Brima et al., SCSL-200416-T, Trial Chamber, Judgement, 20 June 2007. Prosecutor v. Charles Ghanchay Taylor, SCSL03-01-T, 18 May 2012.

80 Por ejemplo, la controversia sobre las acciones de los "freedom fighters" en contextos de conflicto armado. Ver: Cassese, A., ob. cit.

81 Para enfoques doctrinarios sobre un crimen internacional de terrorismo, cfr.: Cassese, A., ob. cit.; Di Filippo, M. (2008). "Terrorist Crimes and International Co-operation: Critical Remarks on the Definition and Inclusion of Terrorism in the Category of International Crimes". European journal of international law, ISSN 0938-5428, Vol. 19, Nro. 3, 533-570, etc. En materia de aportes jurisprudenciales, cabe mencionar la sentencia del TEL, en el que se sostuvo -no sin polémica- que el terrorismo ha devenido en un crimen de Derecho Penal Internacional. Ver: Danessa, S. (julio-agosto 2018). "Reflexiones sobre la definición de Terrorismo. El cuestionado Tribunal Especial para el Líbano". Revista Argumentos, Nro. 6, 12-34; Ambos, K. (enero de 2012). "Creatividad judicial en el Tribunal Especial para el Líbano: ¿es el terrorismo un crimen internacional?". UNED Revista de Derecho Penal y Criminología, $3^{\text {a }}$. Época, Nro. 7, 143-173. 
Prosecutor v. Radislav Krstić

IT-98-33-T, Trial Chamber, Judgment, 2 August 2001.

IT-98-33-A, Appeals Chamber, Judgment, 19 April 2004.

Prosecutor v. Momir Nikolić

IT-02-60/1-S, Trial Chamber, Sentencing Judgment, 2 December 2003.

Prosecutor v. Zejnil Delalić, Zdravko Mucić a / k a "Pavo", Hazim Delić, Esad Landžo a/k/a "Zenga"

IT-96-21-T, Trial Chamber, Judgment, 16 November 1998.

IT-96-21-A, Appeals Chamber, Judgment, 20 February 2001.

Prosecutor v. Zlatko Aleksovski

IT- 95-14/1-T, Trials Chamber, Judgement, 25 June 1999.

IT-95-14/1-A, Appeals Chamber, Judgement, 24 March 2000.

Prosecutor v. Stanislav Galić

IT-98-29-T, Trial Chamber, Judgement, 5 December 2003. Separate and partially dissenting opinion of Judge Nieto-Navia.

IT-98-29-T, Appeals Chamber, Judgement, 30 November 2006. Separate and partially dissenting opinion of Judge Schomburg. Separate Opinion of Judge Shahabuddeen. Separate and Partially diseenting Opinion of Jugde Meron.

Prosecutor v. Dragomir Milošević

IT-98-29/1-T, Trial Chamber, Judgement, 12 December 2007.

IT-98-29/1-A, Appeals Chamber, Judgement, 12 November 2009 and Partly Dissenting Opinion of Judge Liu Daqun.

Prosecutor $v$. Milan Martić

IT-95-11-A, Appeals Chamber, Judgement, 8 October 2008.

Prosecutor v. Vujadin Popović et al.

IT-05-88-T, Trial Chamber, Judgement, 10 June 2010.

IT-05-88-A, Appeals Chamber, Judgement, 30 January 2015.

Prosecutor $v$. Zdravko Tolomir

IT-05-88/2-T, Trial Chamber, Judgement, 12 December 2012.

IT-05-88/2-A, Appeals Chamber, Judgement, 8 April 2015.

Prosecutor v. Radovan Karadžić

IT-95-5/18-T, Trial Chamber, Judgement, 24 March 2016.

Prosecutor v. Jadranko Prlić et al.

IT-04-74-T, Trial Chamber, Judgement, 29 May 2013.

IT-04-74-A, Appeals Chamber, Judgement, 29 Nov 2017, Partially Dissenting, Dissenting Opinions and Declaration of Judge Liu Daqun.

Prosecutor v. Ratko Mladić

IT-09-92-T, Trial Chamber, Judgement, 22 November 2017.

\section{Jurisprudencia del STSL}

Prosecutor v. Moinina Fofana and Allieu Kondewa

SCSL-04-14-T, Trial Chamber, Judgement, 2 August 2007.

Prosecutor v. Alex Tamba Brima et al.

SCSL-2004-16-T, Trial Chamber, Judgement, 20 June 2007. 
Prosecutor v. Charles Ghanchay Taylor SCSL-03-01-T, 18 May 2012.

\section{Doctrina, referencias normativas e información disponible en sitios de internet}

Ambos, K. (enero de 2012). "Creatividad judicial en el Tribunal Especial para el Líbano: jes el terrorismo un crimen internacional?" UNED Revista de Derecho Penal y Criminología, $3^{\text {a }}$. Época, Nro. 7, 143-173.

Cassese, A. (2006). "The Multifaceted Criminal Notion of Terrorism in International Law". Journal of International Criminal Justice 4(5): 933-958.

Danessa, S. (julio-agosto 2018). "Reflexiones sobre la definición de Terrorismo. El cuestionado Tribunal Especial para el Líbano". Revista Argumentos, Nro. 6, 1234.

Di Filippo, M. (2008). “Terrorist Crimes and International Co-operation: Critical Remarks on the Definition and Inclusion of Terrorism in the Category of International Crimes". European Journal of International Law, ISSN 0938-5428, Vol. 19, Nro. 3, 533-570.

Jodoin, S. (2007). “Terrorism as a War Crime”. International Criminal Law Review 7, 77-115.

Paredi, L. (June 2015). "The war crime of terror: an analysis of international jurisprudence”. International Crimes Database (ICD) Brief 11.

Swinnen, J. "Sobre los 'actos de Terrorismo' en el Derecho Internacional Humanitario. Una mirada desde los Convenios y Protocolos de Ginebra". Prudentia Iuris, $\mathrm{N}^{\circ} 86$.

S.C. Res. 808, 1, U.N. Doc. S/RES/808 (Feb. 22, 1993).

S.C. Res. 827, U.N. Doc. S/RES/827 (May 25, 1993).

S.C. Res. 955, U.N. Doc. S/RES/955 (Nov. 8, 1994).

S.C. Res. 1166, U.N. Doc. S/RES/1166 (May 13, 1998).

S.C. Res. 1315, U.N. Doc. S/RES/1315 (Aug. 14, 2000).

S.C. Res. 1329, U.N. Doc. S/RES/1329 (Nov. 30, 2000).

S.C. Res. 1411, U.N. Doc. S/RES/1411 (May 17, 2002).

S.C. Res. 1431, U.N. Doc. S/RES/1431 (Aug. 14, 2002).

S.C. Res. 1481, U.N. Doc. S/RES/1481 (May 19, 2003).

S.C. Res. 1597, U.N. Doc. S/RES/1597 (April 20, 2005).

S.C. Res. 1660, U.N. Doc. S/RES/1660 (Feb. 28, 2006).

S.C. Res. 1837, U.N. Doc. S/RES/1837 (Sept. 29, 2008).

S.C. Res. 1877, U.N. Doc. S/RES/1877 (July 7, 2009).

S.C. Res. 1931, U.N. Doc. S/RES/1931 (June 29, 2010).

S.C. Res. 1993, U.N. Doc. S/RES/1993 (June 29, 2011).

S.C. Res. 2306, U.N. Doc. S/RES/2306 (Sept. 6, 2016).

https://www.icty.org/sid/24 (disponible al 9/8/2020). 